\title{
A Feasibility Study of a Telehealth Intervention on Health Care Service Utilization among Transgender Women of Color in Washington, DC
}

\author{
Manya Magnus ${ }^{1}$ Elizabeth Edwards ${ }^{2}$ Aurnell Dright ${ }^{2}$ Leandrea Gilliam² Angela Brown ${ }^{2}$ \\ Matthew Levy ${ }^{1}$ Neal Sikka ${ }^{3}$ Marc Siegel ${ }^{4}$ Vittoria Criss ${ }^{2}$ Christopher Chauncey Watson ${ }^{2, *}$ \\ Edward Machtinger $^{5}$ Irene Kuo ${ }^{1}$
}

\footnotetext{
${ }^{1}$ Department of Epidemiology and Biostatistics, Milken Institute School of Public Health, The George Washington University, District of Columbia, United States

${ }^{2}$ Contractors at the Department of Epidemiology and Biostatistics, Milken Institute School of Public Health, The George Washington University, District of Columbia, United States

${ }^{3}$ Section of Innovative Practice, Department of Emergency Medicine, The George Washington University, District of Columbia, United States

${ }^{4}$ Medical Faculty Associates, The George Washington University, District of Columbia, United States

${ }^{5}$ Department of Medicine, University of California, San Francisco, California, United States
}

ACI Open 2018;2:e1-e9.
Address for correspondence Manya Magnus, PhD, MPH, Department of Epidemiology and Biostatistics, Milken Institute School of Public Health, The George Washington University, 950 New Hampshire Avenue, NW, Washington, DC 20052, United States (e-mail: manyadm@gwu.edu).

\begin{abstract}
Keywords

- telehealth

- telemedicine

- transgender women

- HIV

Background Transgender women of color (TWC) are a medically underserved population who often experience substantial barriers to care. TWC experience high rates of stigma, violence, and entrenched barriers to receiving routine or specialty health services. Novel ways to improve access for TWC are urgently needed. Telehealth is one way to support TWC in overcoming barriers, yet this approach has been largely unexamined. The purpose of this study was to develop a TWC-specific telehealth intervention to increase access to primary and specialty care and then pilot test this intervention in a sample of TWC with at least one structural barrier to care.

Methods Eligible participants were 18 years or older, identified as male sex at birth with a current gender identity of either female or transgender, a member of a racial/ ethnic minority, and had experienced at least one study-defined structural barrier to primary or specialty care in the past 6 months. Following a 3-month preintervention phase, participants began a 3-month telehealth intervention which provided secure, remote access to trained, culturally appropriate, peer health consultants (PHCs) via video chat, e-mail, text, or phone. Health care utilization was assessed monthly via computer-assisted self-interview. Outcomes of intention to seek care in the next month and receipt of care in the past month were modeled using generalized estimating equations (GEE).
\end{abstract}

Mr. Watson was an employee of George Washington University during the conduct of the study and preparation of the publication, and is now an employee of Gilead Sciences. received

February 13, 2018

accepted

February 13, 2018
DOI https://doi.org/

$10.1055 / \mathrm{s}-0038-1639603$.

ISSN 2566-9346. (c) 2018 Georg Thieme Verlag KG Stuttgart · New York
License terms

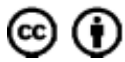


Results Twenty-five eligible participants were enrolled in the study; a majority were black (96\%), older than 25 years (69\%), living with human immunodeficiency virus (HIV) (52\%), and reported depressive symptomatology (67\%). Of the 16 who had at least one pre- and one intervention data collection point, 13 downloaded the mobile video chat application and 7 participated in a qualitative exit interview. The intervention was associated with significantly $(p<0.05)$ increased odds of intention to seek transgender-specific care (adjusted odds ratio, aOR: 1.76 [95\% confidence interval, Cl: 1.0013.08]); participants with depression defined by an elevated Center for Epidemiologic Studies 8-item depression scale (CES-D-8) score were significantly more likely to have intention to seek specialty care (aOR: 10.53 [95\% Cl: 1.42-77.97]), HIV-specific care (aOR: 2.56 [95\% Cl: 1.27-5.17]), and mental health care (aOR: 2.56 [95\% Cl: $1.27-$ 5.17]) during the intervention period. Participants with elevated CES-D-8 scores had significantly greater odds of having sought HIV-specific care (aOR: 2.31 [95\% Cl: 1.314.06]) during the intervention period relative to those with lower scores.

Conclusion These pilot data suggest that telehealth with remote access to PHCs who can provide immediate, culturally competent, nonclinical, education, and referral guidance may be effective in overcoming multiple barriers and improving care utilization outcomes for TWC. Telehealth may be an innovative, low-cost solution to improve health outcomes for populations with multiple barriers to health care services.

\section{Background}

Transgender women (TW) are a medically underserved and largely understudied population who often experience barriers to care. ${ }^{1-10}$ This population experiences high rates of stigma, violence, and an inability to receive even the most basic health care; this is particularly true with the subpopulation of TW of color (TWC) (African American, Native American, Latina, and/or Asian Pacific Islander). ${ }^{11}$ TWC are among those most highly affected by human immunodeficiency virus (HIV), recent and past trauma and violence, stigma, discrimination, and other barriers to primary and specialty care. ${ }^{12-14}$ The need for credible, sensitive, and comprehensive health care for TWC has been identified in numerous studies, ${ }^{1-14}$ yet unfortunately, lack of access to care and culturally competent clinicians continue to serve as structural barriers to health-related services.

Despite profound decreases in HIV infection over the past decade, Washington, DC, still has one of the highest HIV/AIDS rates in the United States, with a pronounced epidemic among minority populations. ${ }^{15}$ Through $2016,1.9 \%$ of the population was living with HIV, with $3.1 \%$ prevalence among black persons and $4.4 \%$ among black men who have sex with men (MSM). District of Columbia has not only a generalized HIV epidemic but also co-occurring concentrated epidemics among MSM and persons who inject drugs. Health disparities are particularly evident with $81.6 \%$ of all cases among black or Hispanic/Latino persons; among transgender persons living with HIV, 96.5\% were persons of color. Transgender persons of color were more likely to present at first laboratory testing with Stage 3 disease than their white or Latino/Hispanic counterparts (35 vs. 27 and 14\%, respectively). Recent needs assessment data ${ }^{16}$ reveal that TWC in
District of Columbia reflect the numerous challenges to health and health care receipt shown in other studies. ${ }^{1-14}$

Novel ways to improve access to care among TWC are urgently needed. Telehealth interventions offer new approaches that may be able to provide TWC with care that can overcome the barriers they often experience, yet this approach has been previously unexamined among this population. Telehealth generally refers broadly to the use of telecommunication technologies and electronic information to provide remote clinical and nonclinical services to patients where telemedicine refers specifically to the use of telecommunication technologies to clinically examine, diagnose, or provide medical treatment to participants. ${ }^{17}$ For the purposes of this study, we use the broader telehealth approach to embrace a broad range of health care and ancillary services that more closely aligns with the needs of the TWC community. Telehealth allows highly trained and culturally appropriate personnel to provide support, referrals, and serve as a bridge to services. ${ }^{18}$ Such approaches have been successful in providing care for other populations including those with chronic illnesses, provision of specialty care such as dermatology, HIV care, cardiology, and psychiatry, chronic disease, and provision of services in correctional settings. ${ }^{19-25}$ Peer health support or peer health navigation, the linkage of patients with either trained support staff, near peers, or persons who themselves have undergone similar health conditions, have been shown in each of these areas to improve patient outcomes. The purpose of this study was to develop a TWC-specific telehealth intervention to increase access to primary and specialty care and then pilot test the feasibility of the intervention and preliminary outcomes in a sample of TWC with at least one structural barrier to care. 


\section{Methods}

\section{Description of the Intervention}

Based on formative data collection and community input described elsewhere, ${ }^{26}$ a telehealth intervention was developed to increase intention to access primary and specialty health care services (primary aim) and increased selfreported service receipt (secondary aim). Due to the short length of follow-up (3 months preintervention that was compared with 3 months on the intervention), intention to seek care was selected as the primary aim. The telehealth intervention allowed real-time access to a Peer Health Consultant (PHC) through texting, e-mailing, telephoning, and secure video access on a broad range of participant-directed topics $^{26}$ and was based on a virtual PHC model that is consistent with other telehealth modalities that have been effective in both primary and specialty care provision. ${ }^{19-25}$ PHCs from the District of Columbia area with extensive experience working with the TWC community and familiar with their unique health and ancillary health needs were hired and trained on the intervention. Training and oversight included management of the licenses, computer, and methods to teach participants to use the software, documentation of contacts, how to contact physicians and study principal investigator in urgent situations, and codification of referral process. The PHCs worked closely with the study project director and research associates to ensure consistently of documentation and approach for referrals as needed.

Licenses for Digigone, ${ }^{27}$ a secure, HIPAA compliant two-way video chat application useable on iPhone, Android, desktop, and laptop devices were purchased for use during the intervention phase along with gift checks for participants to expand their data usage plans if needed. The intervention included 24hour real-time access to a PHC via text, e-mail, phone, or Digigone on their personal devices with any question or health care referral needs. In this intervention, need-based contact was initiated by the participants while regular check-ins were made by the PHCs during day time hours if there had been contact less frequently than once a month. No clinical care was provided through this intervention; the PHCs provided educational information, referrals, and ancillary care services only. Two physicians served as study investigators and were available 24 hours a day for consultation with the PHCs and to address participant urgent medical concerns.

\section{Measures}

Instrumentation for baseline and monthly follow-up questionnaires was based on existing sources specific to TWC populations wherever possible; where such validated tools were not available, common data collection instruments were adapted for the TWC population. Key stakeholders reviewed all instruments for cultural appropriateness and face validity. Domains of the questionnaires included demographic, behavioral, clinical, and health care utilization characteristics, and structural barriers to primary and specialty care receipt. The Center for Epidemiologic Studies 8-item depression scale (CES-D-8) was used to quantify depressive symptomatology at each time point ${ }^{28}$ because this scale is not normed for TWC, we used the median score as the cut point. Questionnaires were developed and implemented using REDCap. ${ }^{29}$ The primary outcome of interest was intention to seek care, where care was broken into categories including primary, specialty, mental health, HIV, or transgender-specific care. The secondary outcome of interest was having sought these categories of care in the past month.

\section{Participants}

Participants were recruited through incentivized peer referral following recruitment of an initial eligible seed identified during the formative phase of the study. Eligible participants were male at birth but currently identifying as female or transgender, 18 years or older, self-identified as black, African American, Latina/Hispanic, Native American, Pacific Islander, or mixed race, indicated experiencing at least one structural barrier to care in the past 6 months defined as: challenges receiving primary, specialty, or transgender health care services due to any structural barrier (including lack of access, transportation, phone, housing instability, experience of stigma at health care services, or incarceration more than 24 hours) or other self-defined barriers to care. Participants were presented with a list of potential barriers to choose from as well as allowing an open-ended examination of potential structural barriers perceived by the participants. Participants were required to have access to a smartphone (minutes were compensated), be fluent in English, live in the District of Columbia area, and provide initial and ongoing informed consent. Following enrollment, participants completed the baseline questionnaire either in the office on a desktop or on the participant's mobile device in a confidential area. Participants were provided with a $\$ 25 \mathrm{gift}$ check plus a transportation voucher for participating in the baseline visit.

\section{Intervention Phase}

Participants were screened and consented in person; all other visits took place remotely unless the participant preferred to come to the clinic. Monthly following enrollment, participants were provided a secure e-mail link to the REDCap questionnaire and when completed, participants could opt to have the $\$ 25$ gift check mailed to them or come to the study clinic to pick it up. There were two distinct nonoverlapping 3-month phases, one preintervention and one intervention phase. Following 3 months of baseline data collection in the preintervention phase, the intervention was deployed followed immediately by three additional monthly postintervention questionnaires. Two computers were provided to community-based organizations that serve TWC locally in case participants were not able to use their own devices, lost their devices, or ran out of data. Qualitative exit interviews using a semistructured interview guide were conducted following completion of the 6-month study. These were analyzed using content analysis to identify key themes.

\section{Statistical Analyses}

Data from the intervention phase of the study were analyzed using information gained from the monthly questionnaires 
and PHC logs of contact. After describing the sample and intervention utilization characteristics, a generalized estimating equation (GEE) approach was used to model the primary and secondary outcomes of interest defined by self-report on the interview (e.g., intention to seek care, use of care services, respectively, unadjusted and adjusted for identified confounders and the intraparticipant correlation over time, using an independent correlation matrix). GEE was selected due to the panel nature of the data, its accommodation of missing data, and its ability to incorporate intraperson correlation over time and thus reduce variability, particularly with the small sample size. A global pre-post indicator was used as a proxy variable for the intervention to assess for overarching impact of the PHC. In view of the fact that dose of intervention (e.g., contact with $\mathrm{PHC}$ ) is completely determined by the participant, this approach minimized attributing intervention successes to baseline participant differences and biasing the results. Options for care were defined as "primary care (e.g., checkup, cold/allergies, and flu shot)," "specialty care (e.g., dermatology and surgery)," "transgender-specific care (e.g., hormone therapy, transitioning care, and silicone)," "HIV care (includes testing, treatment, and prevention)," "mental health care," and "other care"; participants could select as many or as few as they wished. Because of the small sample size and the pilot nature of this hypothesisgenerating study, two categories of $p$-values were used to assess potential associations to direct development of future interventions. A priori cut points were established at $p<0.05$ and $0.05<p<0.25$. Stata version 13.0 SE (College Station, Texas, United States) was used for analysis.

\section{Results}

\section{Baseline Characteristics}

Twenty-five eligible participants were enrolled in the study; a majority were black/African American, older than 25 years, living with HIV, and had experienced significant challenges such as less than full time work, housing instability, and depressive symptomatology (based on the CES-D-8 cut point), as shown in -Table 1. Despite having common structural barriers to care, relatively high proportions of participants had indicators of strong social support ( - Table 1 ), health insurance, and HIV testing histories. That said, retention remained a challenge as expected: nine (36\%) participants did not complete the study and were excluded from GEE analysis. Of the 16 (64\%) who were retained in the study with at least one pre-and one intervention data collection point, 8 (32\%) took all six questionnaires and 7 participated in the qualitative exit interview. There were not significant $(p<0.05)$ differences in those who did and did not stay in study, though the small sample size prohibited meaningful information in examining attrition.

\section{Types of Support Provided}

All participants interacted with the PHCs during the intervention phase at least once, though the content of interactions varied considerably. Participants interacted with PHCs in a user-driven format, reaching out to the PHCs in the medium of their choosing and discussing the content that
Table 1 Baseline characteristics of participants in telehealth pilot study $(N=25)$

\begin{tabular}{|c|c|c|}
\hline & $n$ & $\%$ \\
\hline \multicolumn{3}{|l|}{ Gender $^{a}$} \\
\hline Transgender & 20 & 80.0 \\
\hline Female & 4 & 16.0 \\
\hline Male & 3 & 12.0 \\
\hline \multicolumn{3}{|l|}{ Race } \\
\hline Black/African American & 24 & 96.0 \\
\hline Hispanic/Latina & 1 & 4.0 \\
\hline \multicolumn{3}{|l|}{ HIV status } \\
\hline HIV positive & 11 & 44.0 \\
\hline HIV negative & 10 & 40.0 \\
\hline Declined to state ${ }^{b}$ & 4 & 16.0 \\
\hline \multicolumn{3}{|l|}{ Age $(y)$} \\
\hline $18-25$ & 5 & 20.0 \\
\hline$>25$ & 11 & 44.0 \\
\hline Declined to state $^{\mathrm{b}}$ & 9 & 36.0 \\
\hline \multicolumn{3}{|l|}{ Employment $^{a}$} \\
\hline Looking for work & 7 & 28.0 \\
\hline Full time $(\mathrm{d})$ & 4 & 16.0 \\
\hline Part time $(\mathrm{d})$ & 4 & 16.0 \\
\hline Currently in school & 3 & 12.0 \\
\hline \multicolumn{3}{|l|}{ Living status $^{\mathrm{a}}$} \\
\hline $\begin{array}{l}\text { House or apartment, rents } \\
\text { or owns }\end{array}$ & 6 & 24.0 \\
\hline Family or friend's home & 11 & 44.0 \\
\hline $\begin{array}{l}\text { Transitional housing or } \\
\text { homeless }\end{array}$ & 4 & 16.0 \\
\hline Other & 4 & 16.0 \\
\hline \multicolumn{3}{|l|}{ Housing stability $^{a}$} \\
\hline Moved in the last $6 \mathrm{mo}$ & 11 & 44.0 \\
\hline $\begin{array}{l}\text { Number of different places } \\
\text { stayed in the last } 6 \text { mo } 3+\end{array}$ & 9 & 36.0 \\
\hline \multicolumn{3}{|l|}{ Depressive symptomatology } \\
\hline CES-D-8 (valid = 21) & \multicolumn{2}{|c|}{$\begin{array}{l}8.86 \text { (SD 6.48) } \\
\text { Range } 0-21 \text {, median } 8\end{array}$} \\
\hline CES-D $>$ score of 8 & 14 & 66.7 \\
\hline \multicolumn{3}{|l|}{ Available social support } \\
\hline $\begin{array}{l}\text { Have family members who } \\
\text { live within an hour's drive } \\
\text { on whom you could rely on } \\
\text { for help in a medical } \\
\text { situation }\end{array}$ & 15 & 60.0 \\
\hline $\begin{array}{l}\text { Anybody who would go to a } \\
\text { medical appointment with } \\
\text { you }\end{array}$ & 15 & 60.0 \\
\hline $\begin{array}{l}\text { Anybody you know who } \\
\text { would lend you } \$ 100 \text { or } \\
\text { more if you needed it }\end{array}$ & 6 & 24.0 \\
\hline
\end{tabular}


Table 1 (Continued)

\begin{tabular}{|c|c|c|}
\hline & $n$ & $\%$ \\
\hline \multicolumn{3}{|l|}{ Health insurance status ${ }^{a}$} \\
\hline Has health insurance & 23 & 92.0 \\
\hline Medicaid & 16 & 64.0 \\
\hline Medicare & 4 & 16.0 \\
\hline Other & 5 & 20.0 \\
\hline \multicolumn{3}{|l|}{ Access to care ${ }^{a}$} \\
\hline $\begin{array}{l}\text { No insurance at least once } \\
\text { in the last } 6 \text { mo }\end{array}$ & 3 & 12.0 \\
\hline $\begin{array}{l}\text { Foregone care at least } \\
\text { once in the last } 6 \text { mo }\end{array}$ & 3 & 12.0 \\
\hline \multicolumn{3}{|c|}{ Types of care received in the last $3 \mathrm{mo}^{\mathrm{a}}$} \\
\hline Primary care & 10 & 40.0 \\
\hline HIV care & 10 & 40.0 \\
\hline Mental health care & 9 & 36.0 \\
\hline $\begin{array}{l}\text { Transgender-specific care } \\
\text { (by licensed provider) }\end{array}$ & 3 & 12.0 \\
\hline $\begin{array}{l}\text { Transgender-specific care } \\
\text { (by unlicensed provider) }\end{array}$ & 2 & 8.0 \\
\hline \multicolumn{3}{|c|}{ Intention to obtain care in next $3 \mathrm{mo}^{\mathrm{a}}$} \\
\hline Primary care & 9 & 36.0 \\
\hline Mental health care & 8 & 32.0 \\
\hline Transgender-specific care & 7 & 28.0 \\
\hline Specialty care & 4 & 16.0 \\
\hline $\begin{array}{l}\text { Offered HIV test at last visit if } \\
\text { HIV negative or unknown } \\
(n=23)\end{array}$ & 10 & 43.5 \\
\hline
\end{tabular}

Abbreviations: CES-D-8, Center for Epidemiologic Studies 8-item depression scale; HIV, human immunodeficiency virus; SD, standard deviation.

${ }^{a}$ Check all that apply. $n=20$ participants identified as transgender; $n=1$ participant checked male, female, and transgender.

barticipants had to be older than 18 years but did not have to disclose their date of birth or HIV status.

met their needs. Topics focused on referrals into care, particularly transgender specialty care and mental health services. There was a range of consultation provided, however, from emotional support, hearing from the PHCs about their own experiences, to practical and specific medical information such as when to get screened for HIV.

Baseline attitudes and perceptions regarding health care are described in -Table 2. Despite the eligibility criteria limiting the sample to those who have at least one structural barrier to care, the majority of participants agreed or strongly agreed with statements indicating that they knew how to obtain health care services both emergently and routinely, and levels of provider trust were relatively high. Although participants perceived that their transgender-specific transitioning needs were not always met, they did perceive that such needs could be met by both clinical and nonclinical personnel, which was reassuring in light of the chosen intervention. Baseline descriptive data suggest that participants encountered structural and other barriers to care, but were not extremely distanced or alienated from health care settings.

\section{Acceptability and Uptake of Telehealth Intervention}

Prior to the study, only 7 (29\%) participants reported they had ever used a mobile health application to support health-related behavior, but 20 (83\%) participants were willing to download such an application (data not shown in table). Participants reported they would be more willing to download a mobile health application if recommended by a health care provider (88\%) than a friend or peer (75\%). Reflecting this baseline acceptance, all participants who remained in study until the intervention was deployed engaged in the intervention in at least one way (e.g., text, e-mail, and phone). Thirteen of the 16 (82\%) participants who entered the intervention phase downloaded the Digigone video chat application, all onto Android devices. However, despite having access to the face-to-face contact provided by this application, no participants used it; only texting, e-mailing, and phone contacts occurred.

\section{Primary Outcomes}

- Table 3 describes characteristics associated with the intervention (intervention phase vs. preintervention phase). Examination of potential confounders and effect modifiers suggested that CES-D-8 was a key confounder with independent variables and all outcomes of interest, and was highly collinear with HIV status and age. Additional variables were considered as confounders as well, though the sample size necessarily limited inclusion in the full models examined. Therefore, all odds ratios (OR) are adjusted for CES-D-8 category, modeling the time-dependent covariate as having above the median score, with less than or equal to the median score as the referent category. The median was selected as the cut point for depressive symptomatology as the scale itself has not previously been normed for this population.

\section{Intention to Seek Care in the Next Month}

As shown in - Table 3, after adjusting for CES-D-8 category, the intervention was associated with significantly increased odds of intention to seek transgender-specific care (adjusted OR, aOR: 1.76 [95\% confidence interval, Cl: 1.001-3.08]). Examining the stratified results, participants with depression defined by a CES-D-8 score above the median were significantly more likely to have intention to seek specialty care (aOR: 10.53 [95\% CI: 1.42-77.97]), HIV-specific care (aOR: 2.56 [95\% CI: 1.27-5.17]), and mental health care (aOR: 2.56 [95\% CI: 1.27-5.17]) during the intervention. In the hypothesis generating spirit of this pilot study, weaker associations were also noted: the intervention was associated with reduced intention to seek specialty care and, among those with elevated CES-D-8 scores, increased intention to seek transgender-specific care.

\section{Care Seeking in the Last Month}

After adjusting for CES-D-8 score, the intervention phase was borderline associated with significantly increased odds of 
Table 2 Baseline perceptions regarding care access $(N=25)$

\begin{tabular}{|c|c|c|c|c|}
\hline & $\begin{array}{l}\text { Strongly disagree } \\
\text { or disagree } \\
n(\%)\end{array}$ & $\begin{array}{l}\text { Neutral } \\
n(\%)\end{array}$ & $\begin{array}{l}\text { Strongly agree or } \\
\text { agree } \\
n(\%)\end{array}$ & $n$ \\
\hline $\begin{array}{l}\text { If I need to see a doctor I can figure out } \\
\text { how to get one }\end{array}$ & $8(34.8)$ & $3(13.0)$ & $12(52.2)$ & 23 \\
\hline $\begin{array}{l}\text { I can get a medical appointment for } \\
\text { routine care when I am healthy }\end{array}$ & 0 & $4(17.4)$ & $19(82.6)$ & 23 \\
\hline $\begin{array}{l}\text { I trust the doctors and medical providers } \\
\text { I usually see }\end{array}$ & $4(17.4)$ & $5(21.7)$ & $14(60.9)$ & 23 \\
\hline \multirow{2}{*}{$\begin{array}{l}\text { Health care providers can meet my needs } \\
\text { if I want hormone therapy }\end{array}$} & $4(22.2)$ & $2(11.1)$ & $12(66.7)$ & 18 \\
\hline & Medical provider & $\begin{array}{l}\text { Nonmedical } \\
\text { provider }\end{array}$ & Both & \\
\hline \multirow{2}{*}{$\begin{array}{l}\text { This type of provider would be best able to } \\
\text { help me with my health care needs as a } \\
\text { transgender woman }\end{array}$} & $8(33.3)$ & $2(8.3)$ & $14(58.3)$ & 24 \\
\hline & Never & Sometimes & Always & \\
\hline $\begin{array}{l}\text { Health care providers have met my needs } \\
\text { in transitioning if I have not transitioned } \\
\text { and would like to (if applicable) }\end{array}$ & $4(19.0)$ & $8(38.1)$ & $9(42.9)$ & 21 \\
\hline
\end{tabular}

having sought transgender-specific care in the last month (aOR: 2.15 [95\% CI: 0.99-4.48] $p=0.053$ ) and significantly associated with increased odds of seeking HIV-specific care (aOR: 2.31 [95\% CI: 1.31-4.06] $p=0.004$ ). Here, too, weaker associations were noted: the intervention was associated with reduced reports of seeking mental health care and other health care, and among those with elevated CES-D-8 scores, increased odds of seeking specialty care.

Table 3 Adjusted characteristics associated with primary and specialty care utilization $(N=16)$

\begin{tabular}{|c|c|c|}
\hline $\begin{array}{l}\text { Characteristic } \rightarrow \\
\text { Intention to seek type of care in the } \\
\text { next month } \downarrow\end{array}$ & $\begin{array}{l}\text { Intervention period } \\
\text { (vs. pre-intervention period) } \\
\text { Adjusted OR }(95 \% \mathrm{CI})^{\mathrm{a}}\end{array}$ & $\begin{array}{l}\text { Elevated CES-D-8 } \\
\text { (vs. lower CES-D-8) } \\
\text { Adjusted OR }(95 \% \mathrm{CI})^{\mathrm{b}}\end{array}$ \\
\hline Primary care & $0.91(0.52-1.57)$ & $0.99(0.58-1.70)$ \\
\hline Specialty care & $0.39(0.10-1.59) p=0.188^{c}$ & $10.53(1.42-77.97) p=0.02^{d}$ \\
\hline Transgender-specific care & $1.76(1.001-3.08) p=0.049^{d}$ & $1.52(0.87-2.65) p=0.142^{c}$ \\
\hline $\begin{array}{l}\text { HIV-specific care (prevention or } \\
\text { treatment, as applicable) }\end{array}$ & $0.97(0.53-1.77)$ & $2.56(1.27-5.17) p=0.009^{d}$ \\
\hline Mental health care & $0.63(0.28-1.40)$ & $2.80(1.22-6.43) p=0.015^{d}$ \\
\hline $\begin{array}{l}\text { Characteristic } \rightarrow \\
\text { Sought care in last month } \downarrow\end{array}$ & $\begin{array}{l}\text { Intervention period } \\
\text { (vs. pre-intervention period) } \\
\text { Adjusted OR ( } 95 \% \mathrm{CI})\end{array}$ & $\begin{array}{l}\text { Elevated CES-D-8 } \\
\text { (vs. lower CES-D-8) } \\
\text { Adjusted OR }(95 \% \mathrm{CI})\end{array}$ \\
\hline Primary care & $1.32(0.71-2.46)$ & $0.98(0.54-1.78)$ \\
\hline Specialty care & $1.93(0.58-6.43)$ & $2.55(0.70-9.36) p=0.158^{c}$ \\
\hline Transgender-specific care & $2.15(0.99-4.48) p=0.053^{c}$ & $1.11(0.52-2.40)$ \\
\hline $\begin{array}{l}\text { HIV-specific care (prevention or } \\
\text { treatment, as applicable) }\end{array}$ & $0.98(0.61-1.57)$ & $2.31(1.31-4.06) p=0.004^{d}$ \\
\hline Mental health care & $0.52(0.21-1.27) p=0.153^{c}$ & $2.04(0.92-4.53)$ \\
\hline Other type of health care & $7.25(0.91-58.06) p=0.062^{c}$ & $2.48(0.51-12.02)$ \\
\hline
\end{tabular}

Abbreviations: CES-D-8, Center for Epidemiologic Studies 8-item depression scale; Cl, confidence interval; OR, odds ratio.

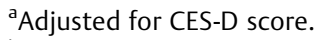

${ }^{\mathrm{b}}$ Adjusted for intervention.

${ }^{\mathrm{c}} 0.05<p<0.25 .{ }^{\mathrm{d}} p<0.05$.

Note: Derived from GEE as described in the methods. 


\section{Qualitative Exit Interviews}

Participants in the exit interviews following the final study assessment reached saturation of theme ${ }^{30}$ in endorsing the telehealth approach used in this study. Participants voiced that they had better access to and were more open to receiving health care services and knew where to seek services due to the PHC and telecommunication with the PHC. In addition, several participants indicated that they were scared or nervous to ask about services or help, but that the telehealth intervention and access to the consultant aided them to overcome this. In addition to making necessary referrals, which participants indicated helped facilitate care, PHCs were said to be able to overcome barriers in processes at health care settings by being able to "get everything on track." Participant choice of mode of communication with the PHC depended largely on their preference and familiarity. Those who were frequently on e-mail or text indicated that was those modalities were easiest for them; people who were more comfortable talking on the phone preferred that modality. The commonality was being able to reach the PHC when there were questions or need for help. All exit interviewees expressed that the accessibility, knowledge base, and affability of the PHCs were key in their ability to improve access to care and overcome challenges they encountered along the way.

\section{Discussion}

This study is the only telehealth intervention to date tailored for the unique needs of TWC and the first to examine the potential for a telehealth intervention using a trained PHC to support TWC to overcome barriers to care. Our findings suggest that this type of intervention is feasible to implement and may effectively facilitate both intention to seek care and increased reports of primary and specialty care sought. The concept of a PHC serving via telecommunication means as a bridge to care is well known to be effective for other specific populations and morbidities; ${ }^{23-25}$ our study suggests that this approach may be highly applicable to TWC population as well. In addition to being modestly associated with improvements in seeking out and having sought out specialty care, data suggest that there may be an impact of the PHC contacts on seeking out more appropriate care. For example, educational conversations may aid TWC in realizing they need transgender-specific health services rather than specialty medical care. This is preliminary in nature only due to the hypothesis-generating nature of the study; future randomized controlled trials and studies with longer observational periods will be needed to fully examine this question.

As we have found in another telehealth study among a larger chronic disease population, ${ }^{31}$ specialized video applications that offer face-to-face viewing may not be required to support patients remotely. Participants connected effectively with PHC staff via less novel telecommunication channels such as text, e-mail, and phone; video contact was not required. Evaluation of exit interviews suggests that this capacity simply was not needed in most cases and that participants felt comfortable communicating in other channels. It may be that the immediacy of contact (24/7 access) with a specific person is more important than viewing; future larger research studies should examine the most operative characteristics of telehealth interventions for TWC to enable development of the most effective approach, ensuring that secure methods are identified that are also easy for TWC to use.

Our findings of differences among TWC suggest that we urgently need new ways to provide care for those women experiencing depression and other related psychological stressors such as trauma, violence, or stigma. Although this study did not have adequate sample size to examine these with more depth, the findings we see are striking in that there is a greater need for services among women with depressive symptomology and that this subgroup may benefit most from telehealth interventions. Our finding regarding care seeking behavior and depressive symptomatology may reflect the increased attention to referrals persons receive from other providers, PHC, or self-referrals, or could also potentially reflect selection bias in our sample. Future studies should examine this outcome more fully in concert with the telehealth intervention.

As with most pilot studies, ours has several limitations. The sample size is necessarily small and within this small group retention was less than optimal due to the many challenges already faced by the TWC population. This limits our ability to adjust for other perhaps important characteristics that may have moderated the relationship between the intervention and the outcomes of interest. While we intended to evaluate a more heterogeneous group of participants with regard to race or ethnicity, most of our participants were black or multiracial, limiting our ability to generalize to other minority populations including Latina women. Due to the importance of keeping the data collection instruments brief, other questions that would be of interest (e.g., detail on services received, sexual and drug use behaviors, trauma, and more complex mental health scales) could not be collected. Here, too, future larger studies may be better able to examine their role in acceptability and impact of the telehealth intervention. Finally, as a nonrandomized study, it is not possible to attribute improvements in outcomes directly to the telehealth intervention. Randomization of the intervention could have potentially alienated the community participating in the study, by giving much needed to support to some and not to others. Stakeholders from the local area felt strongly about this during study development and it was imperative that the study design supported stakeholder needs. However, the use of the pre-/ postdesign allowed a direct comparison of the outcomes following intervention implementation, comparing each person with herself. Finally, our outcome definitions may not be ideal. Intention to seek care is a potential mediating variable between the hypothesized association between the use of the intervention and actually accessing care, and in a short time frame may represent a more proximal effect of the intervention, even if participants had not yet actually accessed care by the end of the intervention period. Our 
loss to follow-up may accentuate this limitation. Future larger and randomized studies will be necessary to more fully evaluate the potential of this telehealth intervention to improve access to care for TW with barriers to care; ideally these will also have a more heterogeneous population of TW, including those of different ethnicities and facing different types of structural barriers. Despite these limitations, this study suggests that there may be multiple downstream impacts of the telehealth intervention and further study is warranted.

This study has several strengths as well. This is the first study to our knowledge to longitudinally examine the impact of a novel telehealth intervention designed specifically with and for TWC with structural barriers to care. While social desirability bias is a key concern in all behavioral studies, the remote data collection reduces this substantially, with participants entering data into a portal rather than through an interview. ${ }^{32}$ The separation of study staff from PHCs reduces the potential for participants to feel obligated to report to study staff or via REDCap biased results or health care seeking behavior. We were surprised by the low uptake of the video chat application, expecting that this secure channel for face-to-face contact would be useful for participants to engage more fully with the staff. The strength of this study is to learn that in fact, less costly and simpler interfaces may be more suitable. If this proves to be the case in future studies, more resources can be devoted to trained PHCs who use traditional modes of communication and less to smartphones or mobile applications. Finally, it is noteworthy that even with the small sample size reduced through attrition of participants and the short duration of follow-up, we were still able to identify several significant findings, underscoring that the potential impact of the telehealth intervention for TWC may have considerable impact.

\section{Conclusion}

In view of the numerous threats to the health of many TWC, identifying new and effective means to improve access to primary and specialty care services is critical. These pilot data suggest that providing immediate remote access to PHCs who can nonclinically advise, answer questions, provide health education, and refer TWC to appropriate, culturally competent care may be effective in overcoming multiple barriers and improving care utilization outcomes. While we collectively work to improve the services provided to TWC who experience structural barriers to care, future randomized studies should expand on this pilot work to explore telehealth as an innovative, low-cost solution to improve health outcomes for TWC.

\section{Clinical Relevance Statement}

Novel ways to improve access to care among transgender women of color are urgently needed. Although telehealth interventions offer new ways to overcome the barriers that transgender women of color often experience, this approach has been previously unexamined among this population. Data from this study suggest that providing immediate remote access to peer health consultants who can nonclinically advise, answer questions, provide health education, and refer patients to appropriate, culturally competent care, may be effective in overcoming multiple barriers and improving care utilization outcomes among transgender women of color.

\section{Conflict of Interest}

None declared.

\section{Protection of Human and Animal Subjects}

All study instruments, interventions, and procedures were approved by the George Washington University Institutional Review Board. None of the faculty or staff have financial interest in or conflict with the vendor providing the Digigone application. Use or nonuse of the application had no impact on staff or participant compensation. No animals were used in this study.

\section{Funding}

This research has been facilitated by the services and resources provided by the District of Columbia Center for AIDS Research, an NIH funded program (AI117970), which is supported by the following NIH Co-Funding and Participating Institutes and Centers: NIAID, NCI, NICHD, NHLBI, NIDA, NIMH, NIA, FIC, NIGMS, NIDDK, and OAR.

This study was funded by a grant from NIH/NIMHD (5R21MD008624). The content is solely the responsibility of the authors and does not necessarily represent the official views of the $\mathrm{NIH}$.

\section{Acknowledgments}

The authors wish to thank the community members, community advisory board, participants, and providers that were a part of this study. In addition, thanks to Madhu Balachandran, Earline Budd, Cyndee Clay, Ruby Corado, Jenna Ebert, Ayana Elliott, Kyle Gordon, Nikardi Jallah, Blaine Parrish, James Peterson, and Ron Simmons for their support of this project's early development and implementation.

\section{References}

1 Ayala DV, Ibañes GE. Barriers to engaging transgender women in HIV observational research. Am J Public Health 2017;107(06):e9

2 Kussin-Shoptaw AL, Fletcher JB, Reback CJ. Physical and/or sexual abuse is associated with increased psychological and emotional distress among transgender women. LGBT Health 2017;4(04): 268-274

3 Wood SM, Lee S, Barg FK, Castillo M, Dowshen N. Young transgender women's attitudes toward HIV pre-exposure prophylaxis. J Adolesc Health 2017;60(05):549-555

4 Clark H, Babu AS, Wiewel EW, Opoku J, Crepaz N. Diagnosed HIV infection in transgender adults and adolescents: results from the National HIV Surveillance System, 2009-2014. AIDS Behav 2017; 21(09):2774-2783

5 Eaton LA, Matthews DD, Driffin DD, Bukowski L, Wilson PA, Stall RD; POWER Study Team. A multi-US city assessment of awareness and uptake of pre-exposure prophylaxis (PrEP) for HIV prevention among black men and transgender women who have sex with men. Prev Sci 2017;18(05):505-516 
6 Salazar LF, Crosby RA, Jones J, Kota K, Hill B, Masyn KE. Contextual, experiential, and behavioral risk factors associated with HIV status: a descriptive analysis of transgender women residing in Atlanta, Georgia. Int J STD AIDS 2017;28(11):1059-1066

7 Le V, Arayasirikul S, Chen YH, Jin H, Wilson EC. Types of social support and parental acceptance among transfemale youth and their impact on mental health, sexual debut, history of sex work and condomless anal intercourse. J Int AIDS Soc 2016; 19(03, Suppl 2):20781

8 Garofalo R, Kuhns LM, Reisner SL, Mimiaga MJ. Behavioral interventions to prevent HIV transmission and acquisition for transgender women: a critical review. J Acquir Immune Defic Syndr 2016;72(Suppl 3):S220-S225

9 Addis S, Davies M, Greene G, Macbride-Stewart S, Shepherd M. The health, social care and housing needs of lesbian, gay, bisexual and transgender older people: a review of the literature. Health Soc Care Community 2009;17(06):647-658

10 Brennan J, Kuhns LM, Johnson AK, Belzer M, Wilson EC, Garofalo R; Adolescent Medicine Trials Network for HIV/AIDS Interventions. Syndemic theory and HIV-related risk among young transgender women: the role of multiple, co-occurring health problems and social marginalization. Am J Public Health 2012;102(09):1751-1757

11 Nuttbrock LA, Hwahng SJ. Ethnicity, sex work, and incident HIV/ STI among transgender women in New York City: a three year prospective study. AIDS Behav 2017;21(12):3328-3335

12 Eaton LA, Kalichman SC, Price D, Finneran S, Allen A, Maksut J. Stigma and conspiracy beliefs related to pre-exposure prophylaxis (PrEP) and interest in using PrEP among black and white men and transgender women who have sex with men. AIDS Behav 2017;21(05):1236-1246

13 Rebchook G, Keatley J, Contreras R, et al; SPNS Transgender Women of Color Study Group. The transgender women of color initiative: implementing and evaluating innovative interventions to enhance engagement and retention in HIV care. Am J Public Health 2017;107(02):224-229

14 Wansom T, Guadamuz TE, Vasan S. Transgender populations and HIV: unique risks, challenges and opportunities. J Virus Erad 2016;2(02):87-93

15 District of Columbia Department of Health. HIV/AIDS, Hepatitis, STD, and TB Administration. Annual Epidemiology and Surveillance Report: Data through December 2016. Available at: https://doh.dc. gov/publication/2017-hahsta-annual-reports. Accessed June 28, 2017

16 District of Columbia Department of Health. HIV among Transgender Persons in the District of Columbia. HIV/AIDS, Hepatitis, STD, and TB: Data through 2014. Available at: https://doh.dc.gov/ sites/default/files/dc/sites/doh/page_content/attachments/HAHSTA\%20Transgender\%20Supplement\%20-\%20Final\%20format.pdf. Accessed June 12, 2017

17 Ekeland AG, Bowes A, Flottorp S. Effectiveness of telemedicine: a systematic review of reviews. Int J Med Inform 2010;79(11): 736-771

18 Takahashi PY, Hanson GJ, Pecina JL, et al. A randomized controlled trial of telemonitoring in older adults with multiple chronic conditions: the Tele-ERA study. BMC Health Serv Res 2010;10:255
19 Gagnon MP, Lamothe L, Hebert M, Chanliau J, Fortin JP. Telehomecare for vulnerable populations: the evaluation of new models of care. Telemed J E Health 2006;12(03):324-331

20 Hanlon P, Daines L, Campbell C, McKinstry B, Weller D, Pinnock H. Telehealth interventions to support self-management of longterm conditions: a systematic metareview of diabetes, heart failure, asthma, chronic obstructive pulmonary disease, and cancer. J Med Internet Res 2017;19(05):e172

21 Trettel A, Eissing L, Augustin M. Telemedicine in dermatology: findings and experiences worldwide - a systematic literature review. J Eur Acad Dermatol Venereol 2017

22 Jackson D, Roberts G, Wu ML, Ford R, Doyle C. A systematic review of the effect of telephone, internet or combined support for carers of people living with Alzheimer's, vascular or mixed dementia in the community. Arch Gerontol Geriatr 2016; 66:218-236

23 Bashi N, Karunanithi M, Fatehi F, Ding H, Walters D. Remote monitoring of patients with heart failure: an overview of systematic reviews. J Med Internet Res 2017;19(01):e18

24 Shea S, Weinstock RS, Teresi JA, et al; IDEATel Consortium. A randomized trial comparing telemedicine case management with usual care in older, ethnically diverse, medically underserved patients with diabetes mellitus: 5 year results of the IDEATel study. J Am Med Inform Assoc 2009;16(04): 446-456

25 Martínez-Alcalá Cl, Pliego-Pastrana P, Rosales-Lagarde A, LopezNoguerola JS, Molina-Trinidad EM. Information and communication technologies in the care of the elderly: systematic review of applications aimed at patients with dementia and caregivers. JMIR Rehabil Assist Technol 2016;3(01):e6

26 Magnus M, Edwards E, Dright A, et al. Development of a Telehealth Intervention to Promote Care-Seeking among Transgender Women of Color in Washington, DC. Presented at the International AIDS Society meeting; Paris, France; July 2017 (Poster number A-854-0213-04282)

27 Digigone Remote Communication Solutions. Available at: http:// www.digigone.com/. Accessed March 22, 2018

28 Radloff LS. The CES-D scale: a self-report depression scale for research in the general population. Appl Psychol Meas 1977; 1(03):385-401

29 Harris PA, Taylor R, Thielke R, Payne J, Gonzalez N, Conde JG. Research electronic data capture (REDCap)-a metadata-driven methodology and workflow process for providing translational research informatics support. J Biomed Inform 2009;42(02): 377-381

30 Creswell JW, Creswell JD. Research Design: Qualitative, Quantitative, and Mixed Methods Approaches. 5th ed. Los Angeles, CA: SAGE Publications; 2018

31 Magnus M, Sikka N, Cherian T, Lew SQ. Satisfaction and improvements in peritoneal dialysis outcomes associated with telehealth. Appl Clin Inform 2017;8(01):214-225

32 Kissinger P, Rice J, Farley T, et al. Application of computer-assisted interviews to sexual behavior research. Am J Epidemiol 1999;149 (10):950-954 